\title{
Evidence-Based, Alternative Cervical Cancer Screening Approaches in Low-Resource Settings
}

Cervical cancer kills approximately 270,000 women worldwide each year, with nearly $85 \%$ of those deaths occurring in resource-poor settings. ${ }^{1}$ Use of the Pap smear for routine screening of women has resulted in a dramatic decline in cervical cancer deaths over the past four decades in wealthier countries. A key reason for continuing high mortality in the developing world is the shortage of efficient, high-quality screening programs in those regions.

In 1999, five international health organizations came together to create the Alliance for Cervical Cancer Prevention (ACCP). * For the next eight years, with support from the Bill \& Melinda Gates Foundation, the partners worked on a coordinated research agenda aimed at assessing a variety of approaches to cervical cancer screening and treatment (especially ones that may be better suited to low-resource settings), improving service delivery systems, ensuring that community perspectives and needs are incorporated into program design, and increasing awareness of cervical cancer and effective prevention strategies. Several outstanding issues were identified at that time. A general issue was a lack of consensus about the most effective and feasible options for improving cancer screening and treatment. Specific issues included uncertainty about the impact of simple screening methods and a screen-and-treat approach on cervical cancer incidence and mortality; the comparative performance of visual inspection methods of screening-visual inspection with acetic acid (VIA) or Lugol's iodine $\left(\right.$ VILI) ${ }^{\dagger}$-and new methods using human papillomavirus (HPV) DNA testing; the optimal ways to reduce false-positive results from visual inspection methods without producing more false-negatives; and any possible links between the use of cryotherapy and subsequent HIV acquisition.

Recent studies and analyses have answered some of these questions and have validated earlier findings related

\footnotetext{
*The ACCP partners involved in research described in this paper were EngenderHealth, International Agency for Research on Cancer (IARC), Jhpiego, Pan American Health Organization (PAHO) and PATH. In 2008, three new partners joined the Alliance: International Union Against Cancer (UICC), Partners in Health, and Programme of Action for Cancer Therapy/International Atomic Energy Agency (PACT/IAEA). More information on ACCP is available online (http://www.alliance-cxca.org).

+VIA involves inspection of the cervix with the naked eye, one to two minutes after the application of 3-5\% acetic acid (vinegar) under adequate light. When white areas are visible on the cervix, the test is considered positive. VILI, a similar technique, is performed with iodine rather than vinegar.

‡Early results of partner studies have been previously reported (source: Tsu V and Pollack A, Alliance for Cervical Cancer Prevention: shifting the paradigm, International Journal of Gynecology \& Obstetrics, 2005, 89 (Suppl. 2):(S1-S59).
}

to safe, effective, operationally feasible and culturally appropriate strategies for secondary prevention of cervical cancer. $^{\ddagger}$ On the basis of these new data and the results of earlier research conducted in 20 African, Asian and Latin American countries, the ACCP partners have summarized and shared key findings and recommendations for effective cervical cancer screening and treatment programs in low-resource settings, as follows.

\section{FINDINGS}

-In low-resource settings, the optimal age-group for cervical cancer screening to achieve the greatest public health impact is 30-39-year-olds. Screening is considered optimal when the smallest amount of resources is used to achieve the greatest benefit. To determine the optimal age for cervical cancer screening, ACCP researchers used two methodologies: modeling and field-based study. Goldie et al. ${ }^{2}$ conducted cost-effectiveness modeling comparing screening strategies in five developing countries. Their model predicted that for 35-year-old women screened only once in their life, a single-visit or two-visit approach with the VIA method could reduce the lifetime risk of cervical cancer by $25 \%$ and HPV DNA testing could reduce it by $36 \%$. (In a single-visit approach, women are screened and treated during the same visit, reducing loss to follow-up; in a two-visit approach, women must return for results and treatment at some time after screening.) Screening women twice, at ages 35 and 40, was predicted to reduce lifetime cancer risk by $65 \%$ (with VIA) or $76 \%$ (with HPV DNA testing). The model estimated that the cost per life-year saved with these approaches would be less than each country's per capita gross domestic product, making them highly cost-effective according to standards set by the World Health Organization's Commission on Macroeconomics and Health. ${ }^{3}$

Sankaranayananan et al. ${ }^{4}$ followed more than 49,000 women aged 30-59 in India for seven years after a single round of VIA screening, with treatment provided as indicated, and found that the intervention had the greatest impact among women in their 30s. The overall reductions in cervical cancer incidence and mortality were $25 \%$ and $35 \%$, respectively, for all the women in the cohort, but they were $38 \%$ and $66 \%$ in the $30-39$ age-group. These results suggest that targeting women in their 30 s can achieve the greatest public health benefit. As additional resources become available, programs can consider expanding the agegroup of women who are screened to include those aged 40 or older.

\section{By Jacqueline \\ Sherris, \\ Scott Wittet, \\ Amy Kleine, \\ John Sellors, \\ Silvana Luciani, \\ Rengaswamy \\ Sankaranarayanan \\ and Mark A.Barone}

Jacqueline Sherris is vice president, Global Programs, and Scott Wittet is lead, Advocacy and Communications, Cervical Cancer Programs, both with PATH, Seattle, WA, USA. Amy Kleine is program manager, the Cervical Cancer Prevention Program, Jhpiego, Baltimore, MD, USA. John Sellors is a clinical professor, McMaster University Department of Family Medicine, Ontario, Canada.

Silvana Luciani is a project manager, Unit of Non-communicable

Diseases, Pan American Health Organization (PAHO), Washington, DC. Rengaswamy Sankaranarayanan is head, Screening Group, International Agency for Research on Cancer (IARC), Lyon,

France. Mark A.

Barone is a senior medical associate, EngenderHealth, New York. 
- Although cytology-based screening programs using Pap smears have been shown to be effective in the United States and other developed countries, sustaining high-quality cytology-based programs is difficult in low-resource settings. Therefore, in settings where health care resources are scarce, they should be directed toward cost-effective strategies that are more affordable and for which quality can be assured.

A cytology-based screening program-using either conventional cervical cytology (Pap smears) or liquid-based cytology-involves the collection of cell samples from the cervix followed by slide preparation, staining, reading and reporting. The process requires a doctor or nurse to collect the sample; sufficient and consistently available supplies and equipment to collect and process the smears; a cytotechnician to process the sample and read the smears; and a pathologist to confirm the positive readings (and randomly sampled negative ones), oversee the laboratory process and approve the final report. Pap smear results typically take days or weeks to become available, at which time women must be notified, counseled and possibly retested at some interval or referred for additional diagnostics or treatment. The cytology screening process, combined with the delays between screening, provision of test results and ultimate treatment (including necessary repeat visits), are major barriers to the success of cytology-based programs in low-resource settings. Although cytology screening has been introduced in developing countries over the past 30 years-mainly in Latin America and the Caribbean-few programs have reported decreases in cervical cancer incidence similar to those observed in the United States, Europe and Australia, primarily because of the challenges described above. ${ }^{5-7}$

The aim of cytology is to detect the precursors of cervical cancer, termed cervical intraepithelial neoplasia (CIN), * enabling treatment before the progression to cancer. However, cytology has only moderate sensitivity in detecting the more advanced precursors; therefore, regular rescreening is important for the effectiveness of programs using this method. A meta-analysis from 2006 found that the average sensitivity of cytology in Europe and North America for detecting potentially precancerous cervical cells was just 53\%. ${ }^{8}$ A recent analysis of data from several Latin American urban centers found that conventional cytology (Pap smears) had a sensitivity of $53 \%$ (and a specificity greater than $99 \%$ ) for detecting moderate to severe lesions, ${ }^{9}$ while a study in rural Peru found that cytology had a sensitivity of $26 \%$ (and a specificity of $99 \%$ ) for detecting CIN of these grades. ${ }^{10}$ Such results, combined with the documented challenges of implementing and sustaining cytology-based programs, have inspired researchers to seek promising alternatives to Pap smear screening with referral of women with positive results for colposcopy.

*Cervical precancer, or CIN, occurs along a spectrum from mild (CIN 1) to moderate (CIN 2) to severe (CIN 3). If untreated, CIN 3 generally progresses to invasive cancer. Of the three precancerous stages, CIN 2 and CIN 3 are of greatest concern and require immediate follow-up. CIN 1 often clears spontaneously.
- The most efficient and effective strategy for detecting and treating cervical cancer precursors in low-resource settings is to screen using either VIA or HPV DNA testing and then to treat using cryotherapy (freezing). This strategy is optimally achieved in a single visit and can be carried out by competent physicians and nomphysicians, including nurses and midwives. The evidence base in support of alternative screening strategies has expanded significantly over the past few years, benefiting especially from large-scale field studies in India, Latin America, Africa, Thailand and China. These studies have focused on assessing visual inspection strategies, HPV DNA testing and cryotherapy. The data collected suggest that for screening, visual inspection of the cervix with the naked eye after application of acetic acid (VIA) or Lugol's iodine (VILI) has sensitivity comparable to or greater than that of cytology. Both physicians and midlevel providers have used visual techniques, and when these professionals are properly trained and supervised, VIA sensitivity has ranged from $41 \%$ to $79 \% .{ }^{10-15}$ Fewer data are available for VILI, but sensitivity with that technique has ranged from $57 \%$ to $98 \% .{ }^{9,16}$ These visual tests require simple equipment and relatively brief training, and can be performed by midlevel health personnel. Results can be communicated to the patient immediately. Furthermore, when indicated, and if the necessary supplies are on hand, treatment can be provided during the same visit. Given the high rates of loss to follow-up that are common in the developing world, a single-visit approach may markedly increase program effectiveness. However, a limitation of visual tests is that they are not reliable in postmenopausal women because of changes in the transformation zone of the cervix, the area in which precursors of cervical cancer arise.

HPV DNA testing has emerged as a new option for cervical cancer screening. A recent, large study in India found that a program strategy based on a single round of HPV testing was associated with reductions of about $50 \%$ in cervical cancer incidence and mortality, whereas strategies based on a single round of VIA or Pap screening had little, if any, effect on these outcomes. ${ }^{17}$ Although the results related to HPV testing are not surprising, the results related to VIA and Pap testing are because they contrast with those from the earlier study in India ${ }^{4}$ and with experience with Pap screening programs. It is generally accepted that Pap programs have had a significant impact on cervical cancer rates in high-resource settings over the past several decades (with-presumably-the relatively low sensitivity of this method being balanced by repetition of testing many times during a woman's life) and, as mentioned above, a number of studies have suggested that VIA has sensitivity equivalent to or greater than that of Pap tests. The lead investigator of the two studies has noted the challenges of interpreting the disparate results and has observed that the treatment rate among VIA-positive women was much higher in the second study than in the first, which may be a factor in the difference in study results. ${ }^{17}$

HPV DNA tests can use cervical or vaginal samples, often obtained with a brush instead of a swab. The sam- 
ples are collected either by a trained provider or, in the case of vaginal sampling, by the woman herself. * Once collected, the samples can be stored in a preservative solution until testing. The sensitivity of HPV DNA tests for detecting CIN 2-3 ranges from $66 \%$ to $95 \%$, with most studies reporting values greater than $85 \%$ among women aged 30 or older. ${ }^{10,16,18,19}$ These tests are most effective among women at the highest risk for precancerous lesions (i.e., women aged 30 or older) because of the greater likelihood that a positive result at that age signals a persistent HPV infection that could progress to cancer.

Women who test positive for HPV must be further evaluated to determine the best management. In the industrialized world, it is relatively easy to perform colposcopy (visual examination of the cervix with magnification) on HPV-positive women and then treat those found to have histologic evidence of CIN. But this diagnostic step may be difficult to implement in low-resource settings where appropriately trained specialists or necessary equipment is lacking. In such settings, performing VIA or VILI following a positive HPV test is likely to be an important step before treatment to determine whether the cervix has precancerous changes and whether any affected areas are too large or too inaccessible for cryotherapy, and in cases in which cancer is suspected.

In the absence of contraindications, women who are HPV-positive can undergo cryotherapy even if they do not have any visible cervical lesions (especially if the chances are poor that they will return to the clinic for follow-up care), given that such women are at relatively high risk for developing $\mathrm{CIN}^{20}$ This is accomplished by freezing the entire cervical transformation zone, where lesions occur. If cryotherapy is contraindicated, women can be referred for alternate diagnostic and treatment procedures that require colposcopy, such as the loop electrosurgical excision procedure (LEEP) and cold knife conization.

- The use of HPV DNA testing followed by cryotherapy results in a greater reduction in the incidence of cervical cancer precursors than the use of other screen-and-treat approaches. Two key studies have followed women screened using various approaches and treated with cryotherapy to determine their long-term risk of highgrade cervical lesions, cervical cancer or both. In a study conducted in South Africa, 7,000 women were screened with VIA and HPV DNA testing. ${ }^{20}$ Women with positive results were randomized to one of three arms-immediate treatment with cryotherapy following a positive HPV DNA result, immediate treatment following a positive VIA result, or delayed treatment. Efficacy, measured at both 12 and 36 months, showed that the prevalence of CIN 2-3 was much lower in the immediate treatment group, particularly among women screened with HPV DNA testing. At 12 months, the cumulative detection of CIN 2-3 was $1 \%$ among women screened with HPV DNA testing, compared with 3\% among women screened with VIA; the rate in the delayed treatment group was $5 \%$. Hence, in this setting, HPV DNA testing with cryotherapy was more effec- tive than VIA with cryotherapy at reducing the occurrence of more advanced precancerous lesions over time. The trend in detection rates for VIA and HPV DNA testing continued through 36 months of follow-up. ${ }^{21}$ Furthermore, as noted earlier, the most recent data from India show that HPV DNA testing resulted in greater mortality reduction than cytology or VIA screening. ${ }^{17}$

Although HPV DNA testing performs well when compared with other screening tests, commercially available HPV DNA tests such as Hybrid Capture 2 (QIAGEN Inc.) are relatively expensive and involve sophisticated processing in a laboratory; moreover, results become available only after seven hours. Experience to date in low-resource settings suggests that these factors-combined with potential challenges in collecting specimens-limit the applicability of the currently marketed test. Until it is possible to produce a test with similar performance that is simpler to use, less expensive and more robust in typical developing-country conditions, HPV DNA testing is unlikely to reach its full potential in reducing cervical cancer.

Fortunately, a new, rapid HPV DNA test called careHPV is being developed for the market in low- and middle-income countries. ${ }^{19,22}$ It will have a lower cost per test than Hybrid Capture 2 and will be simpler to perform. Moreover, it will be portable and will allow for field interpretation of results within 2.5 hours. The sensitivity and specificity of careHPV were evaluated first in Shanxi, China. ${ }^{19} \mathrm{~A}$ total of 2,500 rural women aged 30-54 were screened, and results from both vaginal and cervical samples were encouraging. The accuracy of the test is substantially better than that of VIA and approaches that of Hybrid Capture 2. The test should reach the market in 2010 or 2011.

Within several years, other molecular and biochemical tests currently undergoing field evaluation could reduce both cost and the delay in obtaining results, making mass testing feasible in low-resource settings.

- When conducted by competent providers, cryotherapy is a safe way of treating precancerous cervical lesions and results in cure rates of at least $85 \%$. Cryotherapy is widely considered to be an effective and appropriate means of treating precancerous cervical lesions. In 2003, the ACCP published a systematic literature review of 38 studies on the safety, effectiveness and acceptability of this therapy. ${ }^{23}$ The results showed an overall cure rate of about $90 \%$. The review concluded that cryotherapy was as effective as other outpatient treatment methods, particularly for treating mild and moderate lesions (CIN 1-2).

Recent studies have supported previous findings on the effectiveness of cryotherapy. The incidence of CIN 2-3 at 12 months after treatment was two-thirds lower among HPV-positive South African women who received cryotherapy than among their counterparts who were assigned to delayed evaluation and treatment. ${ }^{20}$ In addition, Peru's

*Self-sampling has been shown to be less sensitive than sampling by providers, but its advantages are that speculum examination is not needed and acceptability may be higher, leading to better feasibility and population coverage in some settings. 
TATI project (TATI is the Spanish acronym for "screening and immediate treatment") reported that within three years after having cryotherapy, $88 \%$ of women who had had CIN 1-2 and 70\% of women who had had CIN 3 had been cured. ${ }^{24}$ In this study, the therapy was provided by general physicians, but midwives or other midlevel providers have performed the procedure in other settings with similar results. For example, in Dindigul district in India, Sankaranarayanan et al. ${ }^{25}$ found an overall cure rate of $81 \%$ among women with CIN 1 and $71 \%$ among women with CIN 2-3 when cryotherapy was provided by nurses in field clinics.

Rates of complications and side effects for cryotherapy are consistently low. A review of articles reporting on the safety of this therapy found that complications such as severe bleeding and pelvic inflammatory disease were rare. ${ }^{23}$ Similarly, most ACCP studies have shown extremely low rates of serious adverse events requiring hospitalization, with the most common side effects being fever, pain, watery discharge, bleeding and cramping. ${ }^{20,25,26}$ Data from a study conducted by Denny et al. ${ }^{20}$ in South Africa further confirm the safety of this therapy. Among the almost 950 women who underwent cryotherapy after an initial positive HPV DNA or VIA test, only one experienced a serious adverse event (a woman who refused hospitalization for cervical bleeding).

- Unless cervical cancer is suspected, the routine use of an intermediate diagnostic step (such as colposcopy) between screening and treatment is generally not efficient and may result in reduced programmatic success and increased cost. The success of screening programs is reduced when women must make multiple clinic visits, because each additional visit constitutes a possibility that women will be lost to follow-up, particularly in low-resource settings where there are many barriers to accessing care. ${ }^{27,28}$ The single-visit approach, using either visual inspection or rapid HPV DNA testing, saves time for clinicians and therefore makes it possible to reach more women with screening and treatment services. And although there has been concern that the single-visit approach may result in the inappropriate treatment of invasive cervical cancer with cryotherapy, in trials to date, this event has been very rare. ${ }^{21}$

An additional factor supporting the omission of diagnostic colposcopy and biopsy is that the sensitivity of colposcopy-directed biopsy is not perfect. A study using biopsies from all four cervical quadrants in addition to biopsies of any abnormal areas found the sensitivity of colposcopy for detecting CIN 2-3 to be approximately $70-80 \% .^{29}$ Thus, if providers depend on colposcopic detection of disease before treatment is administered, up to 30\% of women with disease will not receive treatment.

In the TATI project in Peru, the proportion of women who received treatment was significantly greater with the screen-and-treat approach than with an approach that involved referral for an intermediate diagnostic step. With the former approach, only $8 \%$ of women referred for immediate cryotherapy did not receive treatment, whereas
$44 \%$ of women referred for colposcopy did not return to the health facility to receive their treatment. For women who accessed a government-provided Pap screening program in the TATI project area, attrition was even higher$75 \%$ of women with abnormal Pap screening results did not complete their diagnosis or treatment. ${ }^{30,31}$

The high recruitment and follow-up rates seen in projects testing the single-visit approach in South Africa, India and Thailand suggest that women were highly satisfied with the services provided. Not only did they opt for immediate treatment when it was indicated and available, but the availability of treatment greatly relieved their anxiety. ${ }^{32}$

\section{DISCUSSION AND CONCLUSIONS}

Evidence on the effectiveness and feasibility of VIA, HPV DNA testing and cryotherapy in a range of developing-country settings supports the assertion that all women, regardless of where they live, should be screened for cervical cancer at least once in their lifetime. Given that programs using affordable and effective screening and precancer treatment tools have now been demonstrated to prevent cervical cancer, every woman should be able to access these tools regardless of where she lives. In resource-poor countries, many health interventions are likely to be in competition for scarce funds; it is therefore important to continue advocating that cervical cancer prevention be a priority. The evidence also confirms the challenges in implementing cytology-based screening programs in many settings, and highlights the value of approaches that can use primary health care staff, particularly nurses, to provide both cervical cancer screening and services for treating precancerous lesions.

The results of research studies and pilot programs provide a compelling case for the safety and effectiveness of cryotherapy in treating many precancerous lesions without an intervening diagnostic step. An immediate offer of cryotherapy makes single-visit approaches to screening and treatment possible. Single-visit approaches that use VIA for screening can be offered now; in addition, new laboratory-independent and less-expensive HPV DNA tests will be available soon, potentially making single-visit approaches with this testing feasible in many low- to medium-resource settings. Such approaches have the potential to increase program effectiveness markedly given the high rates of loss to follow-up that are common in the developing world due to the difficulties women and their families have in obtaining services (related to transport, clinic hours, costs, child care needs and other factors).

Concerns about the overtreatment may occur with single-visit approaches have been weighed against the low morbidity associated with cryotherapy and the overall program benefit that is gained by ensuring higher rates of treatment. Some evidence also suggests that cryotherapy may be protective against the future development of cervical disease among women infected with HPV. Ongoing studies are assessing both this potential protective effect and the hypothetical risks of increased transmission of HIV and other STIs after cryotherapy. 
Although HPV DNA testing provided to women aged 30 or older is the most efficacious screening approach for identifying advanced precancerous lesions, the currently available HPV DNA test cannot be made broadly available in the developing world because of its technological demands, expense and processing time (generally requiring multiple visits for women who need follow-up care). Ensuring that new, rapid HPV tests become available for such settings at low cost is a top priority. ${ }^{19,22,33}$

Given the effectiveness and relative ease of administering cryotherapy, we view this technology as the most promising method for treating $\mathrm{CIN}$ at the primary health care level in most low-resource settings. Yet cryotherapy equipment is not universally available, and concerns have been raised about the quality of some of the equipment that is in use. Strategies must be explored to ensure that adequate numbers of effective, affordable cryotherapy units are available to cervical cancer prevention programs worldwide. $^{34}$

Simply providing new screening and treatment technologies and approaches is not sufficient to ensure uptake and program success. All ACCP work has involved close engagement with women, their partners, communities and civic organizations. ${ }^{32}$ This engagement is an important component of program success, given that many women and their families have limited understanding of cervical cancer or of the availability of simple interventions to prevent it. ${ }^{35-38}$ Ensuring that accurate and understandable information is available, through mechanisms and formats that are culturally acceptable, should be part of any cervical cancer prevention program.

It also is crucial that women, their families and community organizations be consulted about the kinds of services that are most accessible to them, and that program managers actively address any concerns that may discourage use of services. A study in Peru found that women's satisfaction with health facility services was a strong predictor of their use of cervical cancer screening services. ${ }^{39}$ How women are treated when they visit the clinic and the kinds of follow-up and support they receive if they need additional services should be of particular concern. One reason for the high acceptability of services observed in some studies and projects was likely related to the excellent quality of the services offered and the fact that women felt valued and cared for. ${ }^{32}$ Various strategies, including applying formative research to design programs and recruiting stakeholders to form advisory groups, have been used to ensure that programs are aware of and responsive to community needs. 37,40

For maximum impact, programs also require mechanisms for effective training, supervision and continuous quality improvement. Provision of visual screening tests and cryotherapy in particular are relatively new strategies in many countries, and clear, evidence-based training materials are essential when introducing such programs. As more affordable HPV DNA tests become broadly available, training for this testing will be increasingly important.
Blumenthal et al. reported on the training of health care providers to perform both VIA screening and cryotherapy. The consensus was that it was relatively easy to teach general physicians and nurses the requisite skill set. For example, among providers assessed in Thailand, 61\% achieved a score of $85 \%$ or higher on cervical cancer screening with VIA and $75 \%$ achieved a score of $82 \%$ or higher on cryotherapy for treatment of precancerous lesions. In $99 \%$ of the cases, the newly trained provider's assessment of the cervix and decisions on case management agreed with those of the trainer. ${ }^{41}$ Follow-up assessments in Thailand and Ghana showed that providers' skill level remained high regardless of the amount of time elapsed since initial training. ${ }^{42,43}$ That said, trainers have found it more difficult to teach some providers to assess the cervix and make a treatment decision, particularly nurses who are not accustomed to making such decisions. ${ }^{41}$ Finally, posttraining assessment, which is key to ensuring that competence is maintained, and continuous quality improvement that integrates feedback from patients into strategies for strengthening services have been shown to be important for any new service. ${ }^{30,35}$

The international community must consider some important, unanswered questions regarding screening for cervical cancer in low-resource settings, including the following:

- How should screening and treatment recommendations be revised as HPV vaccination becomes more common in low-income countries?

- What are the most appropriate screening and treatment recommendations for HIV-positive women, given the increasing numbers surviving into their $40 \mathrm{~s}$ and $50 \mathrm{~s}$, as access to antiretroviral drugs and services for individuals living with HIV become more widespread?

- How can access to robust and affordable cryotherapy equipment be increased, along with effective training, so that women who need treatment for precancerous cervical lesions routinely obtain it?

The ACCP partners are committed to exploring these issues and to providing stakeholders at all levels with clear guidance on screening and treatment strategies for lowresource settings. They also are exploring how a cervical cancer screening visit can serve as a core building block for a broader menu of health services needed by women in their 30 s and 40 s, for example, screening for cardiovascular disease, breast cancer and diabetes; management of symptoms of menopause; and provision of other services that are largely unavailable in developing countries, but that address increasingly important public health problems.

\section{REFERENCES}

1. Ferlay J et al., GLOBOCAN 2002: cancer incidence, mortality and prevalence worldwide, version 2.0, Lyon, France: International Agency for Research on Cancer, 2004, <http://www.dep.iarc.fr/globocan/ database.htm>, accessed May 16, 2008.

2. Goldie SJ et al., Cost-effectiveness of cervical-cancer screening in five developing countries, New England Journal of Medicine, 2005, 353(20):2158-2168

3. World Health Organization (WHO), Macroeconomics and Health: 
Investing in Health for Economic Development. Report of the Commission on Macroeconomics and Health, Geneva: WHO, 2001.

4. Sankaranarayanan Ret al., Effect of visual screening on cervical can cer incidence and mortality in Tamil Nadu, India: a cluster-randomised trial, Lancet, 2007, 370(9585):398-406.

5. International Agency for Research on Cancer (IARC), Cervix Cancer Screening, Vol. 10, IARC Handbook of Cancer Prevention, Lyon, France: IARC Press, 2004.

6. Dzuba I et al., A participatory assessment to identify strategies for improved cervical cancer prevention and treatment in Bolivia, Revista Panamericana de Salud Pública, 2005, 18(1):53-63.

7. Moodley J et al., Challenges in implementing a cervical screening program in South Africa, Cancer Detection and Prevention, 2006, 30(4):361-368.

8. Cuzick J et al., Overview of the European and North American studies on HPV testing in primary cervical cancer screening, International Journal of Cancer, 2006, 119(5):1095-1101.

9. Sarian LO et al., Evaluation of visual inspection with acetic acid (VIA), Lugol's iodine (VILI), cervical cytology and HPV testing as cervical screening tools in Latin America, Journal of Medical Screening, 2005, 12(3):142-149.

10. Almonte M et al., Cervical screening by visual inspection, HPV testing, liquid-based and conventional cytology in Amazonian Peru, International Journal of Cancer, 2007, 121(4):796-802

11. Belinson J et al., Prevalence of cervical cancer and feasibility of screening in rural China: a pilot study for the Shanxi Province Cervical Cancer Screening Study, International Journal of Gynecological Cancer, 1999, 9(5):411-417.

12. Sankaranarayanan R et al., Accuracy of visual screening for cervical neoplasia: results from an IARC multicentre study in India and Africa, International Journal of Cancer, 2004, 110(6):907-913.

13. Sankaranarayanan R et al., A cluster randomized controlled trial of visual, cytology and human papillomavirus screening for cancer of the cervix in rural India, International Journal of Cancer, 2005, 116(4): $617-623$

14. University of Zimbabwe, JHPIEGO Cervical Cancer Project, Visual inspection with acetic acid for cervical cancer screening: test qualities in a primary-care setting, Lancet, 1999, 353(9156):869-873

15. Megevand E et al., Acetic acid visualization of the cervix: an alternative to cytologic screening, Obstetrics \& Gynecology, 1996, 88(3): 383-386.

16. Sankaranarayanan R et al., A critical assessment of screening methods for cervical neoplasia, International Journal of Gynaecology $\varepsilon$ Obstetrics, 2005, 89(Suppl. 2):S4-S12.

17. Sankaranarayanan R et al., HPV screening for cervical cancer in rural India, New England Journal of Medicine, 2009, 360(14):13851394.

18. WHO, Comprehensive Cervical Cancer Control: A Guide to Essential Practice, Geneva: WHO, 2006.

19. Qiao YL et al., A new HPV-DNA test for cervical-cancer screening in developing regions: a cross-sectional study of clinical accuracy in rural China, Lancet Oncology, 2008, 9(10):929-936.

20. Denny L et al., Screen-and-treat approaches for cervical cancer prevention in low-resource settings: a randomized controlled trial, Journal of the American Medical Association, 2005, 294(17):2173-2181.

21. Wright T, Columbia University, personal communication, Nov. 13 , 2008.

22. Gravitt P et al., Chapter 3: new technologies in cervical cancer screening, Vaccine, 2008, 26(Suppl. 10):K42-K52.

23. Castro W et al., Effectiveness, Safety, and Acceptability of Cryotherapy: A Systematic Literature Review, Seattle, WA, USA: Program for Appropriate Technology in Health, 2003

24. Luciani S et al., Effectiveness of cryotherapy treatment for cervical intraepithelial neoplasia, International Journal of Gynaecology $\&$
Obstetrics, 2008, 101(2):172-177.

25. Sankaranarayanan R et al., Effectiveness, safety and acceptability of 'see and treat' with cryotherapy by nurses in a cervical screening study in India, British Journal of Cancer, 2007, 96(5):738-743.

26. Gaffikin L et al., Safety, acceptability, and feasibility of a single-visit approach to cervical-cancer prevention in rural Thailand: a demonstration project, Lancet, 2003, 361(9360):814-820.

27. Coffey PS et al., Cryotherapy treatment for cervical intraepithelia neoplasia: women's experiences in Peru, Journal of Midwifery $E$ Women's Health, 2005, 50(4):335-340.

28. Lewis KDC et al., PATH, personal communication, June 16, 2009

29. Belinson JL et al., Cervical cancer screening by simple visual inspection after acetic acid, Obstetrics \& Gynecology, 2001, 98(3):441444

30. Luciani S and Winkler J, Cervical Cancer Prevention in Peru: Lessons Learned from the TATI Demonstration Project, Washington, DC: Pan American Health Organization, 2006.

31. Gage JC et al., Follow-up care of women with an abnormal cytology in a low-resource setting, Cancer Detection and Prevention, 2003 27(6):466-471

32. Bradley J et al., Women's perspectives on cervical screening and treatment in developing countries: experiences with new technologies and service delivery strategies, Women and Health, 2006, 43(3):103121

33. Schiffman M and Wacholder S, From India to the world-a better way to prevent cervical cancer, New England Journal of Medicine, 2009, 360(14):1453-1455

34. Mariategui J et al., Comparison of depth of necrosis achieved by $\mathrm{CO}_{2}$ - and $\mathrm{N}_{2} \mathrm{O}$-cryotherapy, International Journal of Gynaecology $\mathcal{E}$ Obstetrics, 2008, 100(1):24-26.

35. Agurto I et al., Involving the community in cervical cancer prevention programs, International Journal of Gynaecology $\&$ Obstetrics, 2005, 89(Suppl. 2):\$38-\$45.

36. Sherris J et al., Chapter 25: education, training, and communication for HPV vaccines, Vaccine, 2006, 24(Suppl. 3):S210-S218.

37. Bingham A et al., Factors affecting utilization of cervical cancer prevention services in low-resource settings, Salud Publica de Mexico, 2003, 45(Suppl. 3):S408-S416.

38. Harries J et al., Preparing for HPV vaccination in South Africa: key challenges and opinions, Vaccine, 2009, 27(1):38-44

39. Winkler J et al., Women's participation in a cervical cancer screening program in northern Peru, Health Education Research, 2008 23(1):10-24.

40. Sherris J et al., Advocating for cervical cancer prevention, International Journal of Gynaecology \& Obstetrics, 2005, 89(Suppl. 2):S46-S54.

41. Blumenthal PD et al., Training for cervical cancer prevention programs in low-resource settings: focus on visual inspection with acetic acid and cryotherapy, International Journal of Gynaecology $\&$ Obstetrics, 2005, 89(Suppl. 2):S30-S37.

42. Sanghvi H et al., Scale-up, Performance and Quality in the Cervica Cancer Prevention Program in Thailand: Results from the Outcomes Research Study, Baltimore, MD, USA: Jhpiego, 2008

43. Sanghvi H et al. Outcomes Research Study on Cervical Cance Prevention and Treatment: Results from Ghana, Baltimore, MD, USA: Jhpiego, 2008

\section{Acknowledgments}

The ACCP was funded by the Bill E Melinda Gates Foundation. The ACCP Secretariat was housed at PATH, Seattle, Washington, USA.

Author contact:jsherris@path.org 